\title{
Slow intermixing of cells during Xenopus embryogenesis contributes to the consistency of the blastomere fate map
}

\author{
RICHARD WETTS and SCOTT E. FRASER \\ Department of Physiology \& Biophysics and the Developmental Biology Center, University of California, Irvine, Irvine, CA 92717 USA
}

\section{Summary}

The relatively consistent fates of the blastomeres of the frog embryo could result from (i) predetermination of the blastomeres or (ii) reproducible morphogenetic cell movements. In some species, the mixing of the cells during development provides a test between these alternative hypotheses. If blastomeres are predetermined, then random intermixing of the descendants with neighbouring cells could not alter their fate.

To follow cell mixing during Xenopus development, fluorescent dextran lineage tracers were microinjected into identified blastomeres at the 16-cell stage. The labelled descendants of the injected blastomeres were followed over several stages of embryogenesis. After gastrulation, the labelled descendants formed relatively coherent groups in characteristic regions of the embryo. By larval stages, most of the labelled descendants were still located in characteristic regions. However, coherence was less pronounced and individual descendants were located in many regions of the embryo. Hence, cell mixing is a slow, but progressive, process throughout
Xenopus development. This is in sharp contrast to the extensive mixing that occurs during the early development of other vertebrates, such as zebrafish and mice.

The slow cell mixing in Xenopus development suggests a simple mechanism for the consistent fates of cleavagestage blastomeres. The stereotyped cell movements of embryogenesis redistribute the largely coherent descendants to characteristic locations in the embryo. The small amount of mixing that does occur would result in variable locations of a small proportion of the descendants; this could contribute to the observed variability of the blastomere fate map. Because cell mixing during Xenopus development is insufficient to challenge possible lineage restrictions, additional experiments must be performed to establish when and if lineage restrictions occur.

Key words: cell mixing, cell lineage, fate mapping, amphibian gastrulation, fluorescent dextran.

\section{Introduction}

One of the central questions of developmental biology concerns the process by which the one-cell zygote gives rise to all of the specialized cell types in the organism. The mechanisms that control the formation of the different cell types fall into two broad categories. One mechanism is that the precursors are predetermined to forming specific cell types, so that only the cell lineage is important in establishing cell fate. For example, cytoplasmic determinants in the ascidian egg specify which cells develop into muscle (Meedel et al. 1987; Uzman \& Jeffrey, 1986). An alternative mechanism is that indifferent cells become committed to specific fates through interactions with neighbouring cells. For example, cell interactions appear to play a major role in the formation of the nervous system (Gimlich \& Cooke, 1983; Slack et al. 1984; Doe et al. 1985). In many cases, experimental results have indicated that both cell interactions and cell lineages contribute to the final differentiated phenotype (Doe et al. 1985; Ferguson et al. 1987). To study the relative contributions of cellular interactions and predetermination, it is necessary first to elucidate the normal cell lineages.

Fate mapping studies in the frog Xenopus laevis have shown that identified blastomeres produce numerous cell types, but the derivatives and their locations tend to be reproducible (Nakamura et al. 1978; Hirose \& Jacobson, 1979; Jacobson \& Hirose, 1981; Gimlich \& Cooke, 1983; Dale \& Slack, 1987; Moody, 1987a,b). These studies have also demonstrated that blastomere fate is somewhat variable; every experimental animal differs slightly from the others in exact positioning of the descendants from an identified blastomere. Reconciling both the consistency of the fate map and its slight variability has proven difficult. Part of this difficulty is due to the fact that fate maps only show the final distribution of the descendants; the maps themselves cannot provide any information about the morphogenetic cell movements that lead to the final state. Details of the morphogenetic movements can offer important clues about the mechanisms underlying development. 
In particular, random cell intermixing can challenge the blastomere lineages and thereby reveal any intrinsic lineage restrictions.

To obtain information about the morphogenetic cell movements during Xenopus embryogenesis, we studied the mixing of cells labelled with fluorescent dextran lineage tracers (Gimlich \& Braun, 1985). The labelled descendants of identified blastomeres were followed at several developmental stages in order to reconstruct a dynamic picture of mixing. During cleavage stages, labelled descendants remained together in a coherent group, but slowly intermixed during embryogenesis. By larval stages, they had clearly intermixed with neighbouring cells. Thus, cell mixing progresses slowly but continuously throughout Xenopus embryogenesis. Most of the mixing takes place after cell fates are likely to have become fixed. The cell mixing is insufficient to challenge the blastomere lineages and conclusions cannot yet be drawn from presently available data about whether Xenopus blastomeres are predetermined.

\section{Materials and methods}

\section{Microinjection}

Xenopus laevis embryos were obtained by artificial fertilization and individual blastomeres were labelled with fluorescent dextran using pressure injection (O'Rourke \& Fraser, 1986). Lysinated fluorescein dextran (LFD) or lysinated

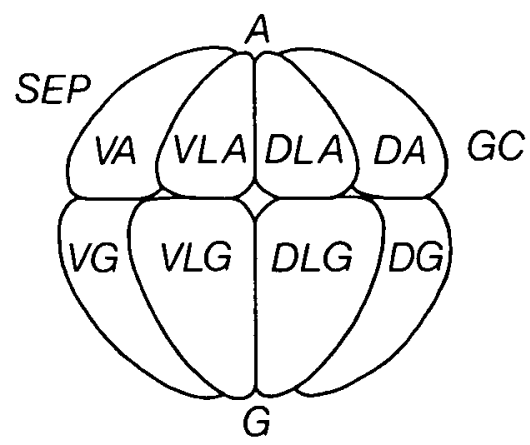

Fig. 1. Identification of blastomeres at the 16-cell stage. This is a view of the left side of the embryo with the animal pole $(A)$ at the top, the vegetal pole $(G)$ at the bottom, the sperm entry point $(S E P)$ at the left, and the grey crescent $(G C)$ at the right. The blastomeres are named using the nomenclature of Gimlich \& Cooke (1983), slightly modified for the 16-cell stage. Dorsal blastomeres are designated with $D$, dorsolateral with $D L$, ventrolateral with $V L$, and ventral with $V$. The animal tier blastomeres end in $A$ and the vegetal cells end in $G$. Thus, blastomere DA (equivalent to D1.1 of Hirose \& Jacobson, 1979) gives rise to two blastomeres of the 32-cell-stage embryo, called D1 and D2 by Gimlich \& Cooke (1983), Ia and Ib by Nakamura et al. (1978), and A1 and B1 by Dale \& Slack (1978). Similarly, blastomere DLA (equivalent to D1.2 of Hirose \& Jacobson, 1979) gives rise to blastomeres $A 2$ and $B 2$ of Dale \& Slack (1987). For this study, we examined animals in which (i) LRD or LFD was injected into either dorsal blastomere of the animal pole (DA or DLA), or (ii) both dyes were injected into adjacent blastomeres. The following figures show animals in which LRD was injected into blastomere DA and LFD was injected into blastomere DLA.
Fig. 2. Positions of labelled descendants in live Xenopus embryos. The left panel of each pair of photographs shows the LRD-labelled descendants of blastomere DA. The right panel shows the LFD-labelled descendants of blastomere DLA in the same animal. Due to the thickness and curvature of these animals, not all of the fluorescence is in the plane of focus. (A) An animal-pole view of a stage 9 embryo. Before gastrulation, labelled descendants form coherent groups in the same relative location as their ancestral blastomere. The solid line indicates the outline of the embryo and emphasizes the large proportion of the blastula without any labelled cells. For orientation, the dotted line indicates the approximate position of the midline; at stage 9 there are no landmarks that indicate accurately the future body axes. From the positions of the labelled clones, the dorsal blastoporal lip should form near the bottom of the figure (cf. Keller, 1975). (B) A dorsal view of a stage 16 neurula; the dorsal midline is indicated by a dotted line. The labelled descendants are still fairly coherent. The DA descendants are located in the rostral region (toward the left) and along the dorsal midline. The DLA descendants are located lateral and posterior to the DA descendants. This animal had some fluorescent debris stuck to its vitelline membrane (arrows). (C) A lateral view of a stage 31 embryo (dorsal midline is at the top). Later in embryogenesis, labelled descendants still occupy the same relative locations as they did in the neurula. The DA descendants (left panel) are located in the rostral region and in the dorsal part of the trunk. The deep structures, the CNS (open arrow) and the myotomes (arrowhead), are out of the plane of focus. The DLA descendants (right panel) are located lateral to the DA descendants. The labelled epidermal cells mostly obscure the deeper labelled cells. Bar, $200 \mu \mathrm{m}$.

rhodamine dextran (LRD; $100 \mathrm{mg} \mathrm{m}^{-1}$; Molecular Probes, Inc.) was pressure injected under the control of a Picospritzer II (General Valve Corp.). The micropipettes were pulled from thin-walled, borosilicate capillary tubing (Frederick Haer, Co.) and were bevelled with $0 \cdot 1$ micron grit metallurgy paper such that a $20 \mathrm{~ms}$ pulse of air expelled approximately $0.5 \mathrm{nl}$ of fluorescent dextran. A total of $2-5 \mathrm{nl}$ of dye was injected into a single blastomere.

After dye injection, the animals were reared (in dilute, artificial pond water) in the dark to minimize bleaching of the fluorescent dye and to avoid phototoxic effects. The majority of injected animals developed normally after dye injection. Animals were rejected from further analysis if: (i) they were grossly abnormal, (ii) individual fluorescent cells were not seen in whole neural-plate-stage animals or (iii) any fluorescent dead tissue, usually located in the digestive tract, was observed in histological sections.

\section{Histology}

In both whole animals and in histological sections, the positions of the labelled cells were noted and the extent of mixing at various stages during embryogenesis was evaluated. Live animals were placed in a dish filled with dilute methanesulphonate anaesthetic before being examined with an epifluorescent microscope (Zeiss Universal) for the distribution of labelled cells throughout the body. After observation, some of the animals were allowed to recover so that they could be re-examined at a later stage. Because the yolk granules contained within the early amphibian embryo scatter the fluorescence from deep cells, only the surface cells could be localized with confidence in whole animals. Conventional 

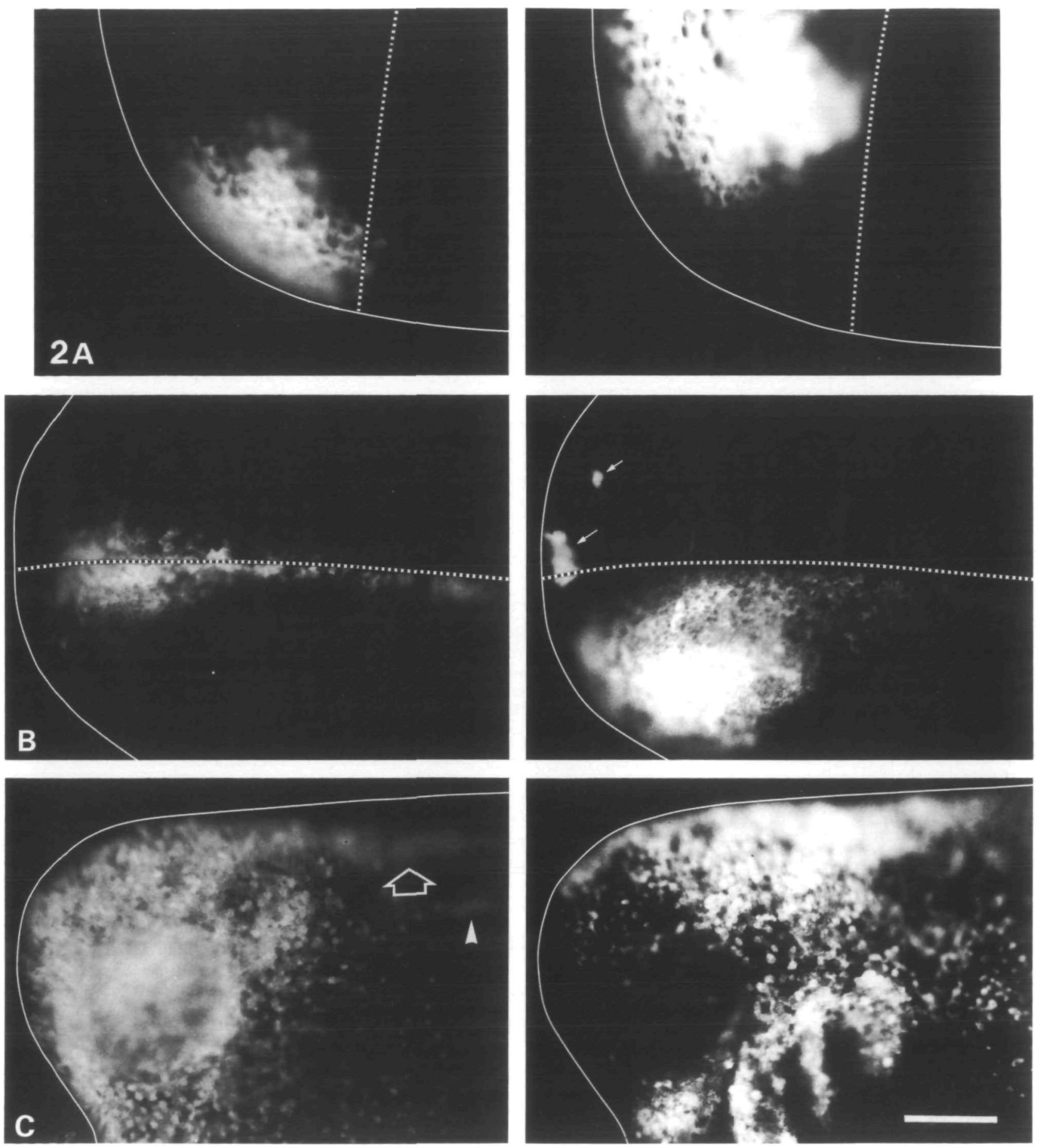

histological techniques were used to follow the descendants located in deeper layers. Animals were fixed with $4 \%$ paraformaldehyde in $0.1 \mathrm{M}$-phosphate buffer ( $\mathrm{pH} 7.4$ ), dehydrated in $95 \%$ ethanol, embedded in methacrylate plastic (LKB Historesin) and serially sectioned at $6 \mu \mathrm{m}$.

\section{Results}

At the 16-cell stage, each blastomere was identified by its position relative to the natural pigmentation of the egg (Fig. 1). Individual blastomeres were injected with

LFD or LRD, and in some animals, two adjacent blastomeres were labelled (one with LFD and the other with LRD). The total volume of the amphibian embryo increases little during embryogenesis, so there is minimal dilution of the lineage tracer. Hence, at the stages that we examined, the descendants of a blastomere labelled with fluorescent dextrans could be clearly identified. We noted the positions of the labelled descendants and evaluated the extent of mixing in over 100 animals at stages $9,16,23,31,39$, and 45 (Nieuwkoop \& Faber, 1967). To obtain an accurate evaluation 
of the amount of mixing, we focused on a tissue that remained continuous throughout these stages and had few physical barriers to the intermixing of the cells: the central nervous system (CNS). The bulk of the CNS is derived from the four dorsal, animal pole, blastomeres (DA and DLA of both sides; Fig. 1). Therefore, we concentrated our observations on the descendants of these blastomeres.

\section{Positions of labelled cells}

At stage 9, prior to gastrulation, labelled descendants formed single coherent groups of cells (Fig. 2A). In the blastula, which is a hollow sphere of approximately 10000 cells organized in five to eight layers, labelled cells were present in all layers from the surface down to the blastocoel. Because the stage 9 blastula lacks landmarks for distinguishing the dorsal-ventral axis, the position of the labelled cells cannot be confidently related to the future body axis.

At neural plate stages (stage 16), labelled cells were located in the rostral region of the animal and extended rostrocaudally along the dorsal midline (Fig. 2B). The area derived from labelling the dorsalmost blastomere (DA) was more rostral and closer to the dorsal midline than the clones formed from more ventral blastomeres (in order: DLA, VLA and then VA). As illustrated in Fig. 3A, the descendants of the DA blastomere were located in the medial neural plate (future ventral CNS), notochord, somites and endoderm. The DLA blastomere contributed cells to the lateral neural plate (future dorsal CNS and neural crest), dorsal epidermis, somites and sometimes endoderm. In the rostral region, labelled descendants of DA were located in the neural plate and epidermis on both sides of the animal's midline.

Descendants from a single blastomere were often distributed to similar regions of all three germ layers. Labelled cells in the mesoderm were immediately subjacent to the labelled cells in the ectoderm (Fig. 3A). Labelled descendants in the endoderm were subjacent to the labelled mesoderm. As in the other germ layers, the endodermal descendants of blastomere DA were located rostral and along the dorsal midline in the trunk, and the DLA descendants were lateral to the DA descendants.

At later stages of development, the majority of the labelled descendants remained localized within the regions described above for the neurula. Fig. 3B shows a late tailbud-stage animal (stage 39 ) in which the DA and the DLA descendants are located in the same tissues as in the neurula shown in Fig. 3A. The proximity of labelled cells across the three germ layers became somewhat disrupted by differential tissue growth and movements. Furthermore, there appeared to be mixing of the labelled descendants. They were primarily located in characteristic regions (e.g. DA descendants in ventral CNS), but individual cells could be found in adjacent areas (e.g. dorsal CNS).

Mixing of cells

In the blastula (stage 9), the labelled descendants
Fig. 3. Distribution of labelled cells in early embryogenesis. (A) A transverse section through the middle of a stage 16 neurula (the dorsal midline is at the top). Labelled descendants are located in characteristic locations. The red DA descendants are located in the medial neural plate ( $n p$, future ventral NS), the notochord $(n)$, the somite $(s)$, and the endoderm $(e)$. The yellow DA descendants are located in the lateral neural plate (future dorsal CNS), the contiguous epidermis $(p)$ and the somite. There is a sharp border between the DA descendants in the medial neural plate and the faintly labelled DLA descendants (marked by arrowheads) in the lateral neural plate. (B) A transverse section through a stage 39 embryo (the dorsal midline is at the top). Late in embryogenesis, the DA and DLA descendants are still located in the characteristic regions described in A for the neurula. A few faintly labelled DLA descendants can be seen in the dorsal neural tube, somite and epidermis. $e$, endoderm; $n$, notochord; $n t$, neural tube; $p$, epidermis; $s$, somite. (C) Section through a stage 9 blastula. The coherence of the labelled descendants includes the deep cells as well as the surface cells. A few individual LRD-labelled cells (arrowheads) appear to be separated from the other DA descendants. (D) Surface view of an intact stage 9 blastula showing mixing of labelled descendants. One LFD-labelled cell (asterisk) is interdigitated with the LRD-labelled cells; it appears yellow due to the red fluorescence from underlying cells. (E) Dorsal view of an intact neurula (stage 16). Rostral is oriented to the bottom of the micrograph. This is the same animal shown in Fig. 2B; the double-exposure photograph juxtaposes the LFD- and LRD-labelled cells and allows an accurate assessment of the intermixing between these two clones. Along the dorsal midline of the trunk (top), there is relatively little mixing at the border between the DA descendants (red) and the DLA descendants (green). There is more mixing in the rostral region of the embryo, but this is difficult to resolve accurately in whole-mount because of light scattering (yellow region). The small arrows indicate fluorescent debris. The open arrow indicates the approximate level of the section shown in F. (F) Transverse section through rostral region of a stage 16 neurula.

Because of the rounded shape of the embryo at this stage, this is a tangential section through the neural plate in an apparently horizontal plane. There is extensive cell mixing in this region, including across the midline (dotted line), with more mixing in the ventral part (bottom). In contrast, there is no mixing in the posterior CNS, as seen in the section of the same animal in $\mathrm{A}$. The asterisk indicates a hole in the plastic section. Bar, $50 \mu \mathrm{m}$ in $\mathrm{A} ; 100 \mu \mathrm{m}$ in $\mathrm{B}, \mathrm{C}$, $\mathrm{D}$ and $\mathrm{F} ; 160 \mu \mathrm{m}$ in $\mathrm{E}$.

formed coherent groups of cells, but the borders between different clones were not absolute. Mixing at the borders resulted in labelled cells interdigitating with unlabelled neighbours (Fig. 3C,D). Individual cells were found up to three cell diameters from other descendants of the same blastomere. This slight mixing at the border between the descendants of two different blastomeres is strikingly different from the extensive intermixing that occurs in zebrafish prior to gastrulation (Kimmel \& Warga, 1986).

At neural plate stages (stage 16), the labelled clones were elongated along the rostrocaudal axis but remained largely coherent. Labelled descendants were 

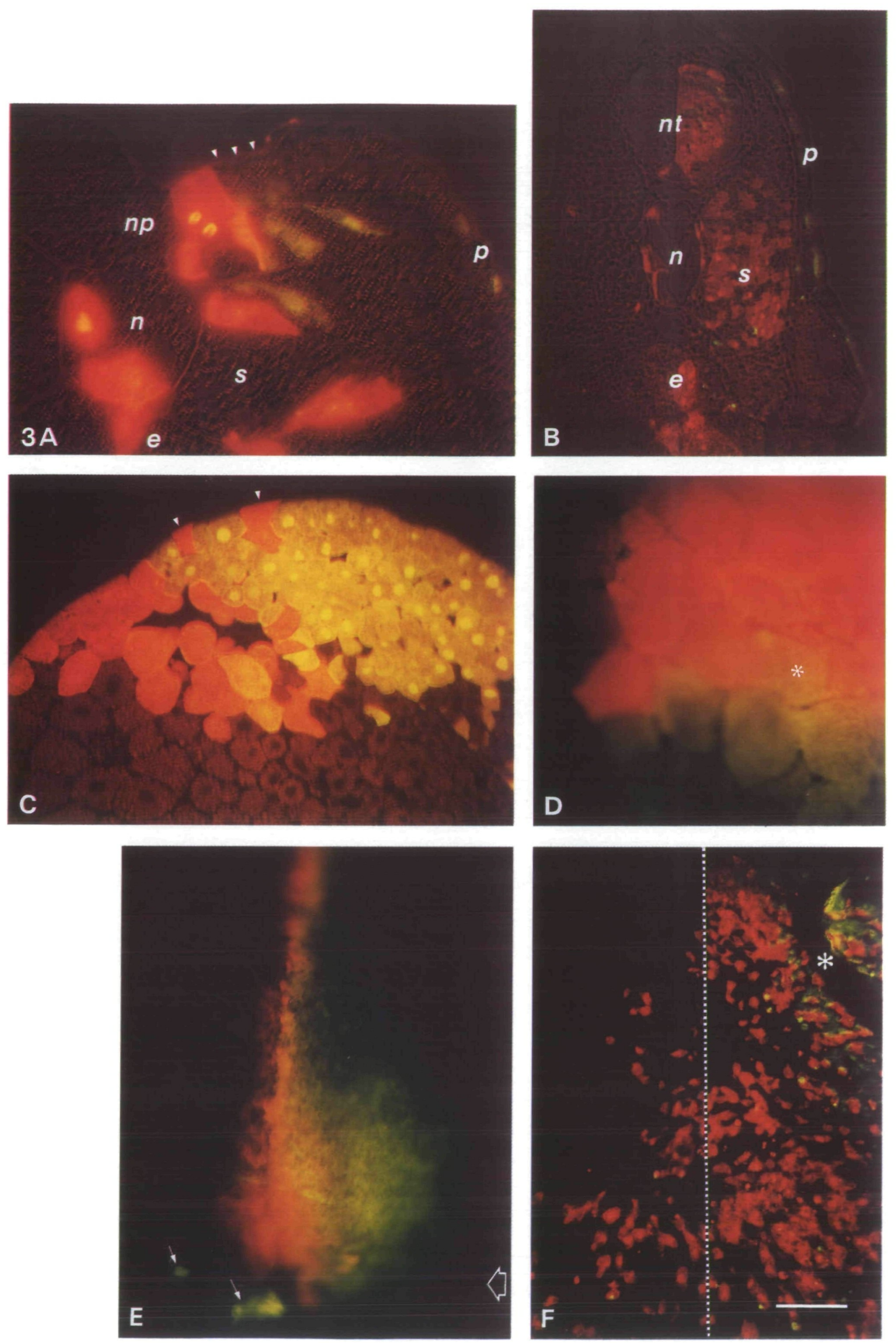

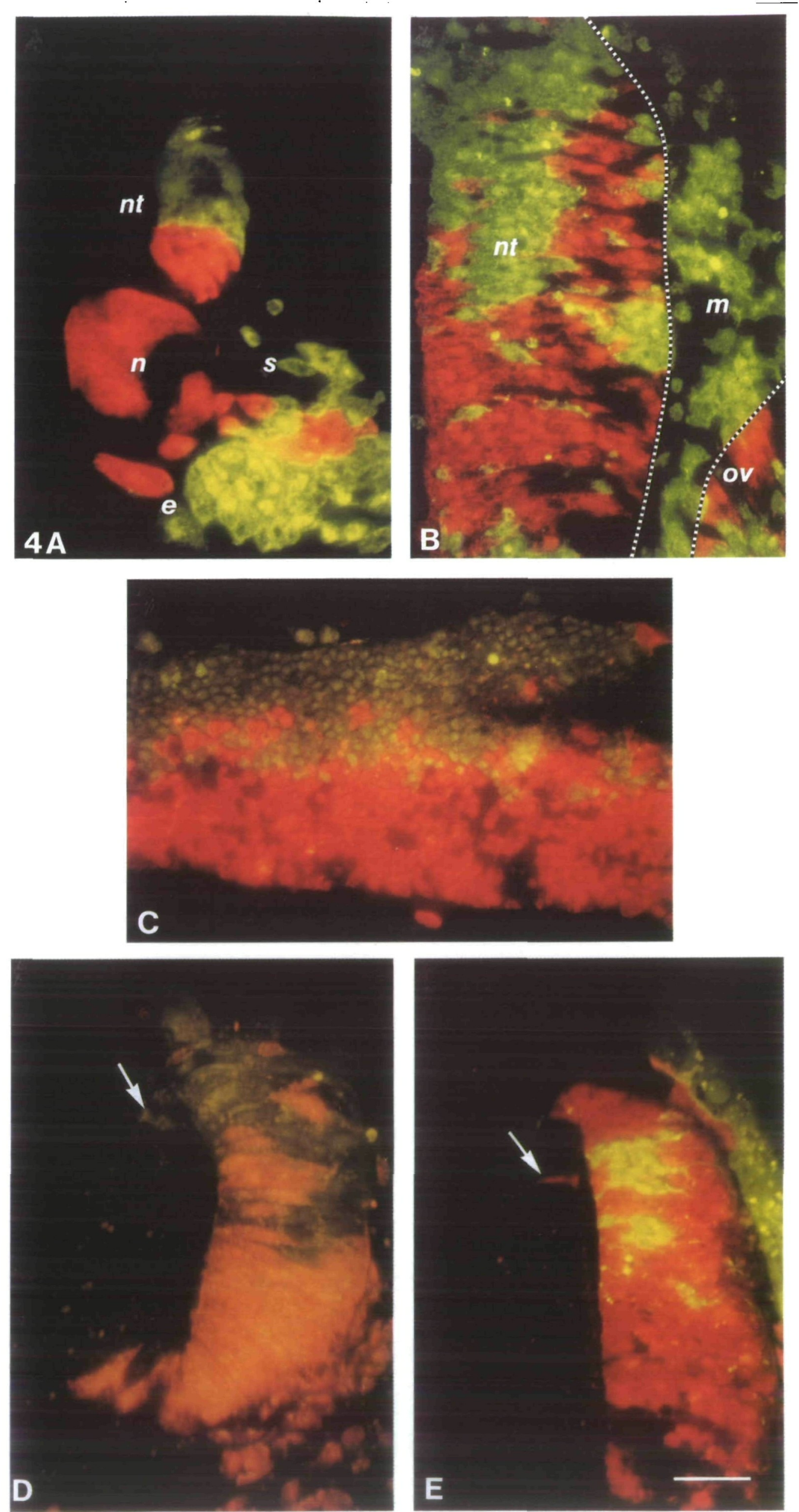
Fig. 4. Distribution of labelled cells in late embryogenesis. (A) Transverse section through the trunk of a stage 23 (neural-tube-stage) embryo. In the CNS, there is a sharp border between the green DLA descendants dorsally (top of micrograph) and the red DA descendants ventrally. $e$, endoderm; $n$, notochord; $n t$, neural tube; $s$, somite. (B) Transverse section through the rostral region of the same stage 23 animal as shown in A. There is extensive mixing of DA and DLA descendants. However, the mixing is not complete; the majority of DA descendants are not interdigitated with DLA descendants and are generally more ventral in location. $m$, mesenchyme; $n t$, neural tube; $o v$, optic vesicle. (C) Parasagittal section through the hindbrain of an early talbud (stage 31) animal. Rostral is toward the right and dorsal is toward the top of the photograph. The green DLA descendants are primarily in the dorsal CNS, with the red DA descendants more ventral, but there is no absolute border. Individual cells or small clusters can be seen on both sides of the border. In the original section, the yellow cells were clearly LFD-labelled DLA descendants. (D) Transverse section through the midbrain of a stage 31 embryo. There is some cell mixing at the border between the green DLA and the red DA descendants. In addition, one descendant of the left DLA blastomere is in the right dorsal CNS (arrow) in this section. At earlier stages, the future dorsal halves of the CNS are physically separated on opposite sides of the neural plate (see Fig. 3A). The green cells in the right half of the CNS in this tailbud-stage animal must have crossed the dorsal midline after neurulation was completed.

(E) Transverse section through the midbrain of a latetailbud-stage animal (stage 39; same animal as in Fig. 3B). Clusters of yellow-green DLA descendants are mixed with the red DA descendants. This animal has numerous DA descendants present in the dorsal CNS as well as in the ventral CNS. Several labelled cells had crossed the dorsal midline; the one visible in this section is marked by the arrow. Dorsal is at the top of all micrographs in this figure. $\mathrm{Bar}, 50 \mu \mathrm{m}$.

usually contiguous along the dorsal midline (Fig. 3E), although some individual cells were separated from the coherent mass of the clone. Fig. $3 \mathrm{~A}$ shows a section through the dorsal trunk of a neurula, demonstrating a sharp border between the descendants of blastomeres DA and DLA. In the more lateral epidermis, individual descendants of the DLA blastomere were mixed with unlabelled cells, probably VLA descendants. In the somites, individual labelled cells were frequently seen, indicating that more mixing had occurred in the mesoderm than in the ectoderm. Gastrulation involves a dramatic rearrangement of the embryo, and the cells in the dorsal region participate in the convergent extension thought to drive gastrulation (Keller et al. 1985; Keller \& Danilchik, 1988). The coherence and elongation of the clones suggests that random cell movements are small in comparison to the stereotyped convergent extension movements.

During gastrulation, the greatest amount of mixing took place in the rostral part of the embryo. As can be seen by comparing Fig. $3 \mathrm{~A}$ and Fig. 3F, the descendants in the trunk of the embryo remained much more coherent than those in the rostral region (Fig. 3F shows the rostral region of the animal in Fig. 3A). Individual cells had mixed extensively with neighbouring cells and could be as much as six cell diameters away from other labelled descendants at the edge of the clone. Cells in the rostral region are not thought to undergo convergent extension (Keller et al. 1985). Hence, regions that differ in types of morphogenetic cell movements also differ in the amount of cell mixing during gastrulation.

In the neural-tube-stage embryo (stage 23), the amount of cell mixing was greater than that seen in the neurula, but the coherence of the clones was still quite striking. In the CNS of the trunk, descendants of a single blastomere formed sharp borders with adjacent clones (Fig. 4A). Some mixing was evident, but usually individual labelled cells were close to a coherent group of cells with the same label. In the rostral CNS, the labelled descendants were much less coherent (Fig. 4B). Because the cells in this region were somewhat mixed in the neurula, it was difficult to decide whether they had mixed further between these stages.

At tailbud stages (stage 31 and stage 39), the labelled clones were somewhat coherent. As can be seen in Fig. 4C, the two largely segregated populations of labelled descendants had mixed only near their margins. Many labelled cells in the CNS were seen in small patches or clusters (Fig. 4C,D,E). These clusters were probably daughter and granddaughter cells that had remained together, while more distantly related cells had mixed to a greater extent. In general, the amount of cell mixing was greater at stage 39 than at stage 31 .

In the early larva (stage 45), labelled descendants were located in specific regions, but completely coherent groups were rarely seen. Most of the CNS had differentiated by this stage. Cell bodies were rounder than at earlier stages, with more space between cells and with many cell-sparse areas consisting of neuropile or fibre tracts. This differentiation contributed to the disruption of coherent groups of labelled cells. In addition, observations of double-labelled animals confirmed that significant cell mixing had contributed to the disruption of the formerly coherent groups. Individual cells or small clusters were mixed with cells labelled with a different lineage tracer. Distinct borders between groups of labelled descendants were infrequent, even in the spinal cord. While the intermixing was profound, it was not complete; even at stage 45 , the majority of the descendants were located in characteristic regions of the animal.

\section{Discussion}

In the experiments presented here, fluorescent dextran lineage tracers were used to follow the descendants of identified blastomeres in the Xenopus larva. The eventual fates of the labelled cells in the larva were essentially identical to those observed by previous investigators using HRP as the lineage tracer (Hirose \& Jacobson, 1979; Moody, 1987a). However, we have extended these fate mapping analyses by noting the positions of the fluorescently labelled descendants and 
evaluating the extent of mixing between the descendants of different blastomeres at several stages of embryogenesis. Before gastrulation, labelled descendants formed a coherent group. After gastrulation, labelled cells were still largely coherent and distributed in predictable locations in the embryo. Throughout development, the descendants of blastomere DA were located in the rostral region and along the dorsal midline in the trunk, and the DLA descendants were located lateral to the DA cells. However, as development proceeded, individual cells had progressively spread into neighbouring regions. Thus, the results show that cell mixing occurs in a slow but continuous process during Xenopus embryogenesis.

\section{Cell mixing and consistency of blastomere fate in other species}

Blastomere fate in invertebrates (nematodes, sea urchins, leeches, insects) is highly reproducible. Frequently this reproducibility is due to the presence of intrinsic lineage restrictions. However, many 'invariant' lineages require cell-cell interactions in order to produce the same cell types in every individual (Shankland \& Weisblat, 1984; Doe et al. 1985; Slack, 1985; Priess \& Thomson, 1987; Kenyon, 1988). In each of these cases, it was only through experimental manipulation that the importance of cell interactions was demonstrated. In normal development, the absence of random cell mixing reliably positions the same cells in the same places, resulting in apparently deterministic fates from a set of non-deterministic cell interactions.

In vertebrates other than amphibians, the fate of identified blastomeres is highly indeterminate. A major source of this variability may be the extensive cell intermixing during development (Mullen, 1977; Herrup et al. 1984; Kimmel \& Warga, 1986; Winkel \& Pedersen, 1988). For example, the descendants of identified zebrafish blastomeres are contiguous until the early gastrula stage (Kimmel \& Law, 1985). Random mixing then distributes the labelled cells throughout the fish embryo before gastrulation (Kimmel \& Warga, 1986). Since labelled cells are scattered throughout the embryo, they contribute to a large variety of structures. The numerous cell types produced by the descendants of individual blastomeres demonstrate that the blastomeres are not determined. Thus, the extensive cell mixing in the zebrafish embryo serves as a natural test of the commitment of the cell lineages to a specific fate.

\section{Slow cell mixing and consistency of blastomere fate in Xenopus}

Based on our observations of fluorescently labelled descendants during Xenopus development, we propose that, as in some invertebrates, limited cell mixing contributes to the consistency of blastomere fate. According to this hypothesis, descendants of a single blastomere are not committed to specific fates before gastrulation. However, the largely coherent descendants of the blastula are distributed by stereotyped cell movements to specific regions of the neurula. Because they are located in predictable portions of the embryo, later developmental events channel the descendants of given blastomeres into predictable fates. Hence, the consistency of the fate map results not from early imposed fate restrictions, but instead from stereotyped morphogenetic movements. The limited amount of mixing observed during embryogenesis (especially during gastrulation) results in variability in the location of a small percentage of descendants. This may contribute to the minor variability in blastomere fates (Moody \& Jacobson, 1983; Moody, 1987a).

\section{The ancestral cell group hypothesis and blastomere fate in Xenopus}

Jacobson $(1982,1985)$ has proposed that seven ancestral cell groups (ACGs), specified at the 512-cell stage, give rise to specific regions of the CNS ('compartments'). The key characteristic of ACGs is that cells intermix extensively within each compartment, but they do not cross the boundaries of that compartment. As experimental support, the labelled descendants of 512-cellstage blastomeres are found to be located within a small area at tailbud stages, presumably part of a single compartment. Many of our observations are compatible with the ACG hypothesis. We observed sharp borders between descendants of different blastomeres in approximately the same locations as the proposed compartment boundaries. Because our data indicate that there is initially little or no mixing, apparent boundaries would be expected. Hence, borders between labelled clones cannot be taken as evidence of spatial lineage restrictions.

Some of our results are difficult to reconcile with the ACG hypothesis. In particular, many tailbud-stage animals have labelled descendants of the left DLA blastomere present in the right half of the dorsal CNS (Fig. 4D,E). Because the left and right halves of the dorsal CNS are not physically contiguous until after neurulation is completed, it is impossible that such labelled cells were part of the right ACG at the 512-cell stage (stage 16; see Fig. 3A). These labelled cells must have crossed the putative boundary at the dorsal midline some time after stage 23 , an event incompatible with the ACG hypothesis. In contrast, the presence of labelled cells in the contralateral, dorsal, CNS strongly supports our idea that slow cell mixing makes such crossings infrequent, but not impossible.

The observations of Jacobson and his coworkers (cf. Jacobson, 1985) support our slow mixing hypothesis. Labelled clones descended from 512-cell-stage blastomeres have relatively few cells, which are likely to remain together in a small area and are unlikely to cross an arbitrary boundary, because little mixing occurs during development. As illustrated by experiments on invertebrate development, both lack of cell mixing and presegregated fates can explain the consistency of a fate map. Only experimental manipulations that challenge the cell lineages can test between these possibilities and demonstrate when cells become committed to specific fates. In some vertebrates, cell mixing is adequate to challenge the lineages, but in Xenopus embryogenesis, the stereotyped cell movements and the slow cell 
mixing do not challenge them sufficiently. Experimental manipulations that directly challenge blastomere lineages are needed to test whether the ACG hypothesis or the slow mixing hypothesis best explains the consistency of blastomere fates.

This work was supported by grants from the National Science Foundation (BNS 8608356) and the Monsanto Corporation and by a McKnight Foundation Scholar Award (S. E. F.). We thank M. S. Carhart, S. Burgan and S. Olson for their technical assistance. Preliminary results from this work have been previously published (Wetts \& Fraser, 1986; Wetts et al. 1988).

\section{References}

Dale, L. \& SLACK, J. M. W. (1987). Fate map for the 32-cell stage of Xenopus laevis. Development 99, 527-551.

Doe, C. Q., Kuwada, J. Y. \& Goodman, C. S. (1985). From epithelium to neuroblasts to neurons: The role of cell interactions and cell lineage during insect neurogenesis. Phil. Trans. R. Soc. Lond. B 312, 67-81.

Ferguson, E. L., Sterngerg, P. W. \& Horvitz, H. R. (1987). A genetic pathway for the specification of the vulval cell lineages of Caenorhabditis elegans. Nature, Lond. 326, 259-267.

Gimlich, R. L. \& Braun, J. (1985). Improved fluorescent compounds for tracing cell lineage. Devl Biol. 109, 509-514.

Gimlich, R. L. \& CoOKE, J. (1983). Cell lineage and the induction of second nervous systems in amphibian development. Nature, Lond. 306, 471-473.

Herrup, K., Wetts, R. \& Diglio, T. J. (1984). Cell lineage relationships in the development of the mammalian CNS. II. Bilateral independence of CNS clones. J. Neurogenet. 1, 275-288.

Hirose, G. \& Jacobson, M. (1979). Clonal organization of the central nervous system of the frog. Devl Biol. 71, 191-202.

JACOBSON, M. (1982). Origins of the nervous system in amphibians. In Neuronal Development. (ed. N. C. Spitzer), pp. 45-99. New York: Plenum Press.

JACOBson, M. (1985). Clonal analysis and cell lineages of the vertebrate central nervous system. A. Rev. Neurosci. 8, 71-102.

JACoBson, M. \& Hirose, G. (1981). Clonal organization of the central nervous system of the frog. II. Clones stemming from individual blastomeres of the 32- and 64-cell stages. J. Neurosci. 1, 271-284.

Keller, R. \& DanilchiK, M. (1988). Regional expression, pattern and tuming of convergence and extension during gastrulation of Xenopus laevis. Development 103, 193-209.

KelLer, R. E. (1975). Vital dye mapping of the gastrula and neurula of Xenopus laevis. I. Prospective areas and morphogenetic movements of the superficial layer. Devl Biol. 42, 222-241.

Keller, R. E., DanilchiK, M., Gimlich, R. \& Shih, J. (1985). The function and mechanism of convergent extension during gastrulation of Xenopus laevis. J. Embryol. exp. Morph. 89 Supplement, 185-209.
Kenyon, C. (1988). The nematode Caenorhabditis elegans. Science 240, 1448-1453.

Kimmel, C. B. \& Law, R. D. (1985). Cell lineage of zebrafish blastomeres. III. Clonal analyses of the blastula and gastrula stages. Devl Biol. 108, 94-101.

Kimmel, C. B. \& WarGa, R. M. (1986). Tissue-specific cell lineages originate in the gastrula of the zebrafish. Science $\mathbf{2 3 1}$ 365-368.

Meedel, T. H., Crowther, R. J. \& Whittaker, J. R. (1987). Determinative properties of muscle lineages in ascidian embryos. Development 100, 245-260.

Moody, S. A. (1987a). Fates of the blastomeres of the 16-cell stage Xenopus embryo. Devl Biol. 119, 560-578.

Moody, S. A. (1987b). Fates of the blastomeres of the 32-cell-stage Xenopus embryo. Devl Biol. 122, 300-319.

Moody, S. A. \& JACOBSON, M. (1983). Compartmental relationships between anuran primary spinal motoneurons and somitic muscle fibers that they first innervate. J. Neurosci. 3, 1670-1682.

Mullen, R. J. (1977). Site of $p c d$ gene action and Purkinje cell mosaicism in cerebella of chimaeric mice. Nature, Lond 270, 245-247.

Nakamura, O., Takasaki, H. \& Nagata, A. (1978). Further studies of the prospective fates of blastomeres at the 32-cell stage of Xenopus laevis embryos. Med. Biol. 56, 355-360.

Nieuwkoop, P. D. \& Faber, J. (1967). Normal Table of Xenopus laevis (Daudin). Amsterdam: North-Holland.

O'Rourke, N. A. \& Fraser, S. E. (1986). Dynamic aspects of retinotectal map formation revealed by a vital-dye fiber-tracing technique. Devl Biol. 114, 265-276.

Priess, J. R. \& Thomson, J. N. (1987). Cellular interactions in early C. elegans embryos. Cell 48, 241-250.

Shankland, M. \& Weisblat, D. A. (1984). Stepwise commitment of blast cell fates during the positional specification of the $\mathrm{O}$ and $P$ cell lines in the leech embryo. Devl Biol. 106, 326-342.

Slack, J. M. W. (1985). From Egg to Embryo. Determinative events in early development. Cambridge: Cambridge Univ. Press.

Slack, J. M. W., Dale, L. \& Smith, J. C. (1984). Analysis of embryonic induction by using cell lineage markers. Phil. Trans. R. Soc. Lond. B 307, 331-336.

Uzman, J. A. \& Jeffery, W. R. (1986). Cytoplasmic determinants for cell lineage specification in ascidian embryos. Cell. Differ. 18 , 215-224.

WETTS, R. \& Fraser, S. E. (1986). Fate mapping of blastomeres involved in Xenopus neural development: Slow intermixing of cells. Soc. Neurosci. Abstr. 12, 1121.

WetTs, R., O'Rourke, N. A. \& Fraser, S. E. (1988). Vital-dye analyses of neural development and connectivity. In The Making of the Nervous System (ed. J. G. Parnavelas, C. D. Stern, R. V. Sterling), pp. 52-69. Oxford: Oxford Univ. Press.

Winkel, G. K. \& Pedersen, R. A. (1988). Fate of the inner cell mass in mouse embryos as studied by microinjection of lineage tracers. Devl Biol. 127, 143-156. 